\title{
Anti Solar Activity of Costus Speciosus Leaves of Sikkim Himalayas
}

\author{
Prasenjit Mitra ${ }^{1}$, Tanaya Ghosh², Prasanta Kumar Mitra ${ }^{3 *}$
}

\begin{abstract}
${ }^{1}$ Department of Biochemistry, All India Institute of Medical Sciences (AIIMS), Basni Industrial Area, MIA 2nd Phase, Basni, Jodhpur, Rajasthan 342005, India

${ }^{2}$ Department of Medical Biotechnology, Sikkim Manipal University, Sikkim Manipal Institute of Medical Sciences, Gangtok, Sikkim, India

${ }^{3}$ Professor \& Head, Department of Medical Biotechnology, Sikkim Manipal University, Sikkim Manipal Institute of Medical Sciences, Gangtok, Sikkim, India
\end{abstract}

DOI: $\underline{10.36347 / \text { sajp.2020.v09i01.002 }}$

| Received: 05.01.2020 | Accepted: 12.01.2020 | Published: 16.01.2020

*Corresponding author: Prasanta Kumar Mitra

\section{Abstract}

Original Research Article

Since long Costus speciosus (C. speciosus) has been used in different system of medicine for medical treatment. The plant has several pharmacological properties like anti inflammatory, anti oxidant, anti microbial, anti cancer, gastro protective, anti diabetic, anti gastric ulcer, hepato protective etc. But anti solar activity of C. speciosus leaves of Sikkim Himalaya is not known in literature. Aim of the present study was, therefore, to examine anti solar activity of C. speciosus leaves, if any and if so effect of extraction solvents on the activity. Leaves of $C$. speciosus were collected and identified by the taxonomist. Solvent extractions of the leaves were made separately by using ethanol, chloroform, methanol, acetone, benzene, and ethyl acetate. The extracts were separately exposed for absorption of UV ray to a spectrophotometer using UV region. Result showed that all extracts of $C$. speciosus leaves had UV absorption property but ethanol extract had maximum activity. Ethanol extract of $C$. speciosus leaves, therefore, may be further studied for isolation of the active compound responsible for UV absorbing property for its use in preparation of sun screen lotions.

Keywords: Costus speciosus leaves; Solvent extractions; UV absorbing property.

Copyright @ 2020: This is an open-access article distributed under the terms of the Creative Commons Attribution license which permits unrestricted use, distribution, and reproduction in any medium for non-commercial use (NonCommercial, or CC-BY-NC) provided the original author and source are credited.

\section{INTRODUCTION}

C. speciosus (family, Costaceae), found in tropical region of India along roadsides, streams and in wastelands, is an erect perennial herb [1]. Up to an altitude of $1200 \mathrm{~m}$ the plant is also found in moist tropical evergreen forests [2].

C. speciosus has different names. In Bengali and Hindi it is known as keu. The herb has several other names like Paskarmula (Guajarati), Tara (Assam), Channakoova (Malayalam), Kostam (Tamil), Kashmeeramu (Telegu), Kembuka (Sanskrit), Spiral flag (English) etc [3].

In traditional medicine $C$. speciosus is used as anthelmintic, expectorant purgative and stimulant. It is also used in the treatment of rheumatism, diarrhea, dysentery, dyspepsia, skin diseases, cough and cold, pneumonia, dropsy, fever, bronchial asthma, urinary diseases, jaundice, eye and ear infections as well as in snake bite cases [4].

C. speciosus contains many pytochemicals. These are dioscin, methyl protodioscin, $\beta$-sitosterol, methylprotogracillin, $5 \alpha$-stigmast-9 (11)-en-3 $\beta$-ol, $\alpha$-tocopherolquinone, 26-diol and its 22-hydroxy derivatives, camphene, glucose, galactose, gracillin, dihydrophytylplastoquinone, protogracillin, protodioscin, dihydrophytylplastoquinone polyphenols, tannins, flavonoids, camphene, furostenol, ribose, 3-O[ $\alpha$-L-rhaphapyranosyl $(1 \rightarrow 2)-\beta$-D-glucopyranosyl]-26O-[ $\beta$-Dglucopyranosyl]-22 $\alpha$-methoxy-(25R)furost-5en-3 $\beta, \quad 26-O-\beta$-D-glucopyranosyl-(25R)-furost-5-ene$3 \beta$, diosgenin etc. $3-\mathrm{O}-\beta$-Dglucopyranosyl $(1 \rightarrow 3)-\beta$-Dglucopyranoside, 8-hydroxy triacontane-25-one, beta amyrine, methyl triacontanoate, $\alpha$-tocopherolquinone, 24-hydroxytriacontan-26-one, 24-hydroxytriacontan27-one, $\quad 3-\mathrm{O}-[\beta$-D-glucopyranosyl $(1 \rightarrow 4)-\beta$-Dglucopyranosyl]-26-O-( $\beta$-Dglucopyranosyl-22 $\alpha$ methoxy (25R) furost-5-en-3 $\beta$, $\beta$-amyrin, $\alpha$-Humulene, 3-O-[ $\alpha$-L-rhamnopyranosyl $(1 \rightarrow 2)-\beta$-D-

glucopyranosyl]-26-O-[ $\beta-\mathrm{D}$-glucopyranosyl]- $\quad 22 \alpha$ methoxy-(25R) furost-5-en-3 $\beta$, diosgenin etc. were also isolated from different parts of C. speciosus [5, 6].

C. speciosus showed different pharmacological activities like anti diabetic, antipyretic, antifertility, anti bacterial, anti fungal, anti-inflammatory, anti oxidant, anti cancer etc. The plant also possess anticholinestrase, 
antihelminthic, hepatopretective, hypolipidemic and adaptogenic activities [7, 8]. Anti solar activity of $C$. speciosus leaves, however, is not known in literature.

Aim of the present work, however, was to see in vitro anti solar activity of $C$. speciosus leaves of Sikkim Himalayas, if any and if so effect of extraction solvents on the said activity.

\section{MATERIAL AND METHODS \\ Plant Material}

Leaves of $C$. speciosus were collected from the local market during June - July and authenticated by the taxonomist of the department of Botany of the University of North Bengal, Siliguri. A voucher specimen (No.SM-MB-011) was kept in the department of Medical Biotechnology, Sikkim Manipal Institute of Medical Sciences of Sikkim Manipal University, Gangtok, Sikkim, India for future reference.

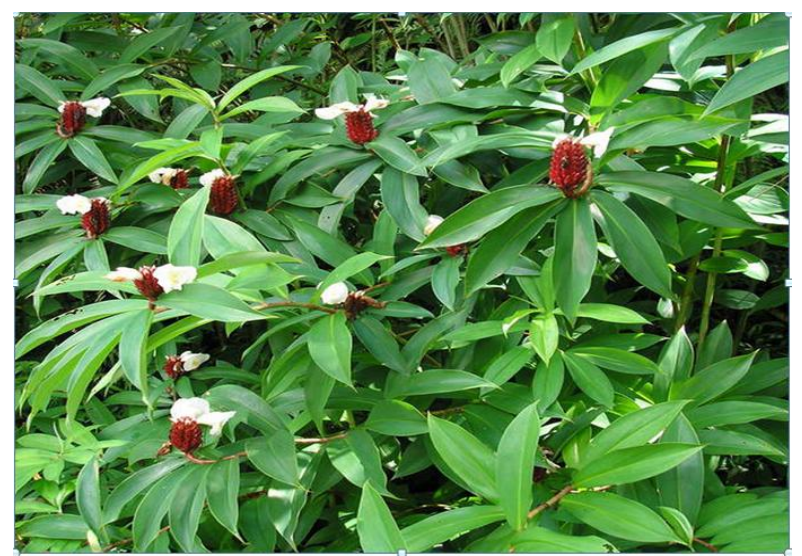

Fig-1: Costus speciosus leaves

\section{Test Drug}

Leaves of $C$. speciosus were washed thoroughly under tap followed by distilled water. Leaves were then shed dried and powered. The powder, used as test drug, was stored desiccated at $4{ }^{0} \mathrm{C}$ until further use.

\section{Solvent Extraction}

Test drug (100g) was extracted separately with $500 \mathrm{ml}$ of ethanol, methanol, acetone, chloroform, benzene and petroleum ether in soxhlet at $37^{\circ} \mathrm{C}$ for 15 minutes. The extract was filtered and the filtrate was evaporated to dryness in vacuo with rotary evaporator at $40-50{ }^{\circ} \mathrm{C}$. This was applied separately for all extracts. Brown masses obtained.

\section{Anti solar activity}

$10 \mathrm{mg}$ of this mass was dissolved in $100 \mathrm{ml}$ distilled water. The solution was processed in a spectrophotometer for UV ray absorption at the range of 200-400 nm.
Chemicals

Chemicals required for the study were purchased from Loba Chem. Lab, Himedia Lab, India and from Merck, Germany.

\section{STATISTICAL ANALYSIS}

All experiments were conducted for three times. Data were analyzed statistically by SPSS 20 . The statistical significance between UV absorption spectra of different extracts was evaluated with Duncan's multiple range test (DMRT). 5\% were considered to be statistically significant [9].

\section{RESULTS}

UV absorption spectra of ethanol, methanol, acetone, chloroform, benzene and petroleum ether extracts of $C$. speciosus Leaves are shown in Figures 2, 3, 4, 5, 6 and 7 respectively.

Ethanol extract of $C$. speciosus leaves absorbs maximum UV ray at $200 \mathrm{~nm}$ (1.50). UV ray absorptions by the same extract at $250 \mathrm{~nm}, 300 \mathrm{~nm}, 350 \mathrm{~nm}$ and 400 nm were $0.97,0.69,0.58$ and 0.49 respectively. At 200 $\mathrm{nm}$ wave length methanol extract absorbs maximum UV rays (1.40). At $250 \mathrm{~nm}, 300 \mathrm{~nm}, 350 \mathrm{~nm}$ and 400 $\mathrm{nm}$ wave lengths ethanol extract of $C$. speciosus leaves showed absorptions $0.79, \quad 0.60, \quad 0.51$ and 0.40 respectively.

Acetone extract of $C$. speciosus leaves showed maximum UV absorption at $200 \mathrm{~nm}$ (1.25). UV ray absorptions by the same extract at $250 \mathrm{~nm}, 300 \mathrm{~nm}, 350$ $\mathrm{nm}$ and $400 \mathrm{~nm}$ were $0.68,0.54,0.47$ and 0.36 respectively. At $200 \mathrm{~nm}$ chloroform extract absorbs maximum UV rays (1.0). At $250 \mathrm{~nm}, 300 \mathrm{~nm}, 350 \mathrm{~nm}$ and $400 \mathrm{~nm}$ wave lengths acetone extract of $C$. speciosus leaves however showed 0.6, 0.43, 0.38 and 0.30 absorptions respectively. Benzene extract showed maximum UV absorption at $200 \mathrm{~nm}(0.90)$. UV ray absorptions by the same extract at $250 \mathrm{~nm}, 300 \mathrm{~nm}, 350$ $\mathrm{nm}$ and $400 \mathrm{~nm}$ were $0.48,0.35,0.30$ and 0.21 respectively. Petroleum ether extract showed maximum UV absorption at $200 \mathrm{~nm}(0.60)$. UV ray absorptions by the same extract at $250 \mathrm{~nm}, 300 \mathrm{~nm}, 350 \mathrm{~nm}$ and 400 $\mathrm{nm}$ were $0.35,0.27,0.24$ and 0.18 respectively.

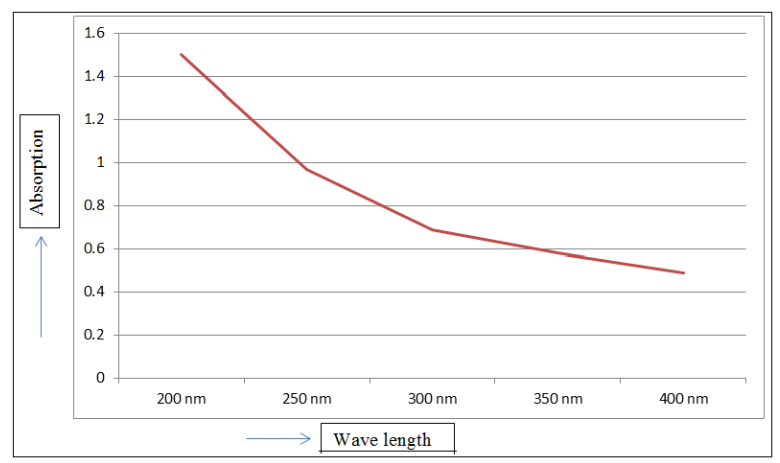

Fig-2: UV radiation absorption by the ethanol extract of $C$. speciosus leaves 


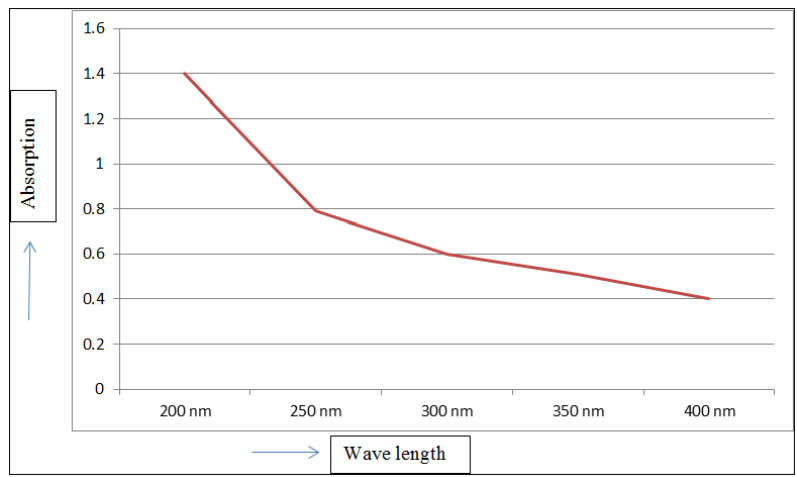

Fig-3: UV radiation absorption by the methanol extract of $C$. speciosus leaves

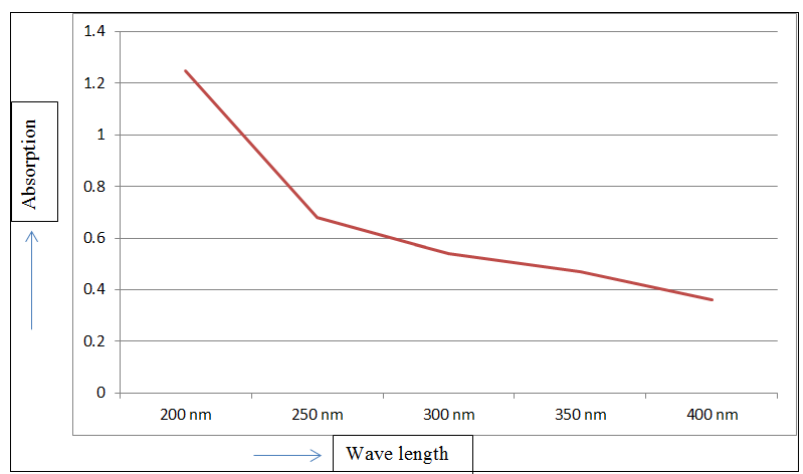

Fig-4: UV radiation absorption by the acetone extract of $C$. speciosus leaves

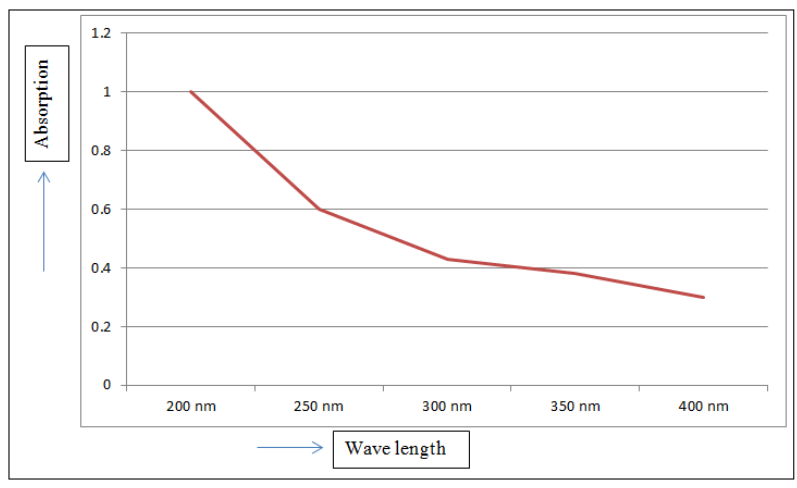

Fig-5: UV radiation absorption by the chloroform extract of $C$. speciosus leaves

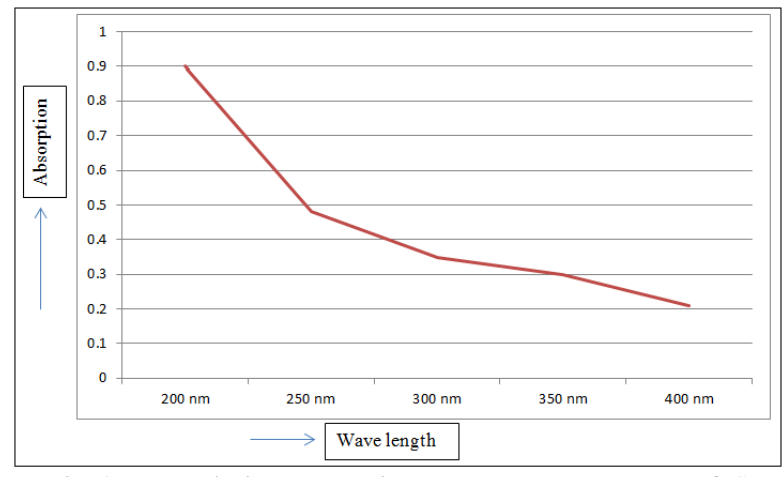

Fig-6: UV radiation absorption by the benzene extract of $C$. speciosus leaves

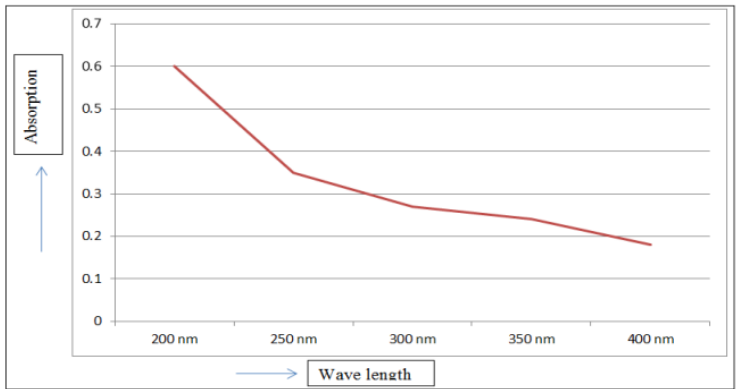

Fig-7: UV radiation absorption by the petroleum ether extract of C. speciosus leaves

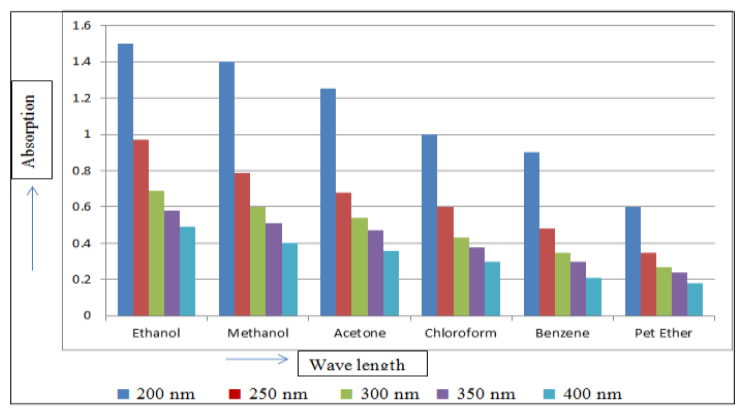

Fig-8: UV radiation absorption at different wave lengths by the ethanol, methanol, acetone, chloroform, benzene and petroleum ether extracts of $C$. speciosus leaves

\section{DISCUSSION}

In electromagnetic spectrum ultraviolet radiation and non-ionizing radiation fall under 180 $400 \mathrm{~nm}$ wavelength region. Ultraviolet radiation may be germicidal, erythermal and black light depending on absorption at wave length $180-280 \mathrm{~nm}, 281-314 \mathrm{~nm}$ and $315-400 \mathrm{~nm}$ respectively. Sunlight is the common source of UV radiation. UV radiation also comes from laboratory equipment like germicidal lamps, cross linker, lasers, Tran's illuminators, and biological safety cabinet [10].

Vitamin D, a nutrient, is very much needed for human health. By absorbing calcium from food and supplements vitamin D helps to maintain strong bones. Cutaneous synthesis of vitamin D depends on solar UVradiation. This covers almost $90 \%$ of the vitamin D requirement of human body. In this way solar UV radiation is good for humans. But solar UV-radiation has bad effect too. It can cause eye and skin injury, stimulate genetically determined photo sensitivities and photosensitivity reactions to ingested drugs. Skin is severely affected if there is over exposure of UV rays. Pigmentary changes atrophy, wrinkling and malignancy may occur. Basal cell carcinoma or malignant melanoma and skin cancer like squamous cell carcinoma may develop. UV radiation also affects eye. Cornea, the outer protective coating of the eye, may be affected. Painful inflammation of eye is seen and if the eye gets chronic UV exposure, then it lead to formation of cataracts. Over-exposure to UV radiation also changes distribution and function of white blood cells in human body. This may cause harmful suppressing effect on the immune system [11]. 
To save humans from ill effect of UV radiation there is continuous search for the sources which can absorb UV radiation from the environment thereby protecting humans. Plants were taken as source and it was found out that many medicinal plants can absorb ultra violet radiation.

Few such medicinal plants are, Azadirachta indica, Aloe vera, Carica papaya, Lycopersicon esculantum, Oscimum sanctum, Mentha piperita, Calotropis gigantean, Phyllostachys pubescens etc [12, 13].

Anti solar activity of $C$. speciosus leaves is not reported in literature. In present study we have shown that $C$. speciosus leaves of Sikkim Himalayas exert anti solar activity. All solvent extracts of $C$. speciosus leaves can absorb ultraviolet radiation. Maximum absorption was found at $200 \mathrm{~nm}$. Further, ethanol extract of the leaves had maximum absorption in all the wave lengths of UV region (Figure-8).

It is known that biological activity of medicinal plants varies with extraction time and temperature [14-17]. We are now interested to see the effect of time and temperature on extraction process to get maximum ultraviolet radiation absorption by $C$. speciosus leaves. Work is going now in our laboratory in this direction.

\section{CONCLUSION}

In the present study we found UV radiation absorption property of $C$. speciosus leaves of Sikkim Himalayas. The property may be utilized in preparation of sun screen lotions to protect humans from UV radiation.

\section{ACKNOWLEDGEMENTS}

We gratefully acknowledge the cooperation of taxonomists of the department of Botany, University of North Bengal, Siliguri, Dist. Darjeeling, and West Bengal for identification of $C$. speciosus leaves.

\section{Conflict of interest: Nil}

\section{REFERENCES}

1. Gupta AK, Tondon N, Sharma M. Quality Standards of Indian Medicinal Plants, Medicinal Plants Unit, Published by Indian Council of Medical Research. 2008; 7:48.

2. Gupta RK. Medicinal and Aromatic Plants, CBS Publishers and Distributors, New Delhi. 2010; 499.

3. Swati S, Agarwal P. Kebuka (Costus Speciosus): A critical review. World Journal Pharm Pharm Sci. 2015;4:421-31.

4. Srivastava Shruti, Singh Pradeep, Mishra Garima, Jha KK, Khosa RL. Costus speciosus (Keukand):
A review. Der Pharmacia Sinica. 2011; 2(1):118128.

5. Rastogi RP, Mehrotra BN. Compendium of Indian Medicinal plants, Central Drug Research Institute, Lucknow and National Institute of Science Communication and Information Resources, New Delhi. 2004; 4:224-225.

6. Gupta MM, Lal RN, Shukla YN. Phytochemicals present in Costus speciosus root. Phytochemistry. 1981; 20(11): 2553-2555.

7. Bhattacharya SK, Parik AK, Debnath, PK, Pandey, VB, Neogy, NC. Anticholinesterase activity activity of Costus speciosus alkaloids. Indian Journal Pharmacol. 1972; 4:178-178.

8. El-Far AH, Shaheen HM, Alsenosy AW, El-Sayed YS, Al Jaouni SK, Mousa SA. Costus speciosus: Traditional Uses, Phytochemistry, and Therapeutic Potentials. Pharmacognosy Reviews. 2018 Jan 1;12(23):120-127.

9. Bliss CI. Statistics in biology, Statistical methods for research in the natural Sciences, Vol. 1, McGraw Hill Book Company, NY. 1967: 558.

10. MacKie RM. Effects of Ultraviolet Radiation on Human Health. Radiation Protection Dosimetry. 2000; 91(1-3):15-18.

11. Gharge VG, Yadav A. Study of Methanolic Extract of Leaves Calotropis gigantean (L.) As an Anti- Solar. Adv Complement Alt Med. 2018; 1(4): 1-3.

12. Gupta D. Absorbing Properties of Some Plant Derived Extracts. Research Journal of Chemical and Environmental Sciences. 2013; 1(2): 34-36.

13. Gupta V. Environment friendly antibacterial and uv protective finish on cotton using $\mathrm{S}$. cumini (1.) leaves extract. International Journal of Textile and Fashion Technology. 2017; 7(1): 53-62.

14. Monton C, Luprasong C. Effect of Temperature and Duration Time of Maceration on Nitrate Content of Vernonia cinerea (L.) Less.: Circumscribed Central Composite Design and Method Validation. International journal of food science. 2019;2019.

15. Sulaiman IS, Basri M, Masoumi HR, Chee WJ, Ashari SE, Ismail M. Effects of temperature, time, and solvent ratio on the extraction of phenolic compounds and the anti-radical activity of Clinacanthus nutans Lindau leaves by response surface methodology. Chemistry Central Journal. 2017 Dec;11(1):54.

16. Wingard MR, Phillips RC. Solvent extraction IV. The effect of temperature on extraction rate. Journal of the American Oil Chemists' Society. 1951;28:149-152.

17. Amir Hamzah NA, Morad NA, Nordin MFM, Ilia Anisa AN, Yusof YAM., Effect of extraction time and temperature on the extraction of phenolic compounds from Orthosiphon stamineus leaves. Aust J Basic \& Appl Sci. 2017; 11(3):15-21. 